www.jmscr.igmpublication.org Impact Factor 5.244

Index Copernicus Value: 83.27 ISSN (e)-2347-176x ISSN (p) 2455-0450 crossref DOI: https://dx.doi.org/10.18535/jmscr/v5i1.05

\author{
Journal Of Medical Science And Clinical Research \\ IGM Publication \\ An official Publication of IGM Publication
}

\title{
Conventional MRI Features of Atypical Meningiomas
}

\author{
Authors \\ Beenamol S, Sharmad M S, Meer Chisthy \\ Medical College Trivandrum Kerala \\ Corresponding Author \\ Beenamol S \\ Associate Professor, Dept of Radiodiagnosis, Medical college Trivandrum Kerala
}

\begin{abstract}
Background: Atypical meningiomas form a small group among meningiomas and are characterised by distinct natural history and outcome. Pre-operative detection of atypical meningiomas can help in streamlining the management.

Methods: This study was done at Government Medical College, Trivandrum over a 3 year period. Study population included the patients with meningiomas sent for Magnetic Resonance Imaging, who were investigated by previous CT scan. Sample size was 183. Study design was Observation study. Final pathological diagnosis in all patients was confirmed by biopsy and histo-pathological examination.

Results: Of the 183 cases studied, 165 cases were typical meningiomas, 15 were found to be atypical and 3 were anaplastic.

Conclusion: MR imaging is sensitive in differentiating between typical and atypical meningiomas and can be used as a problem solving study when there is diagnostic dilemma with CT scan showing indeterminate findings. This makes conventional advanced MRI as the imaging of choice in meningiomas.
\end{abstract}

Key Words: Meningioma; Atypical; MRI Brain tumours.

\section{Introduction}

$75 \%$ of meningiomas are benign and are classified as grade I according to World Health Organization (WHO) standards. According to 2016 WHO classification, subtypes such as atypical, clear cell and choroid are classified as Grade 2 and malignant meningiomas are categorized as grade3.Grades 2 and 3 meningiomas display less favourable clinical outcomes. Atypical meningiomas account for between 4.7 and $7.2 \%$ of all meningiomas. Malignant meningiomas are less common, comprising between 1.0 and $2.8 \%$. Malignant and atypical meningiomas are more prone to recurrence and rapid growth. The distinction between benign and atypical or malignant meningioma is very important, because surgical and treatment planning as well as prognostication will depend on those pathologic types. This study attempted to prospectively elucidate MRI characteristics of atypical meningiomas.

\section{Materials and methods}

183 cases of meningiomas were treated surgically in our hospital from January 2010. Out of these cases 15 cases were histologically identified as atypical meningiomas. The patients comprised of 9 males and 6 females, ranging in age from 25 to 70 years. Neurological symptoms such as 
headache, loss of consciousness, and numbness of the extremities were reported. Duration of symptoms ranged from 1 month to 16 years.

Inclusion Criteria: Patients with CT evidence of Meningioma

Exclusion Criteria: Patients with past history of primary brain tumours (treated with surgery / radiotherapy / both), Patients with systemic conditions preventing adequate study employing all required sequences, Patients unfit for major neurosurgery, Patients with inoperable tumours / patients with H/P report not possible),Patients not willing to participate in the study, Patients with usual contra indications to MR Imaging like cardiac pace maker, cochlear implants and other metallic objects within the body, Patients not giving consent to participate in the study.

\section{Imaging Technique and Protocol}

MRI examinations were performed on 1.5 T MR System, Siemens Magnetom Avan to MRI machine using a phased array head coil. After scout view MRI, the examination protocol consists of pre-contrast $\mathrm{T} 1$ and $\mathrm{T} 2$ fast spin-echo (FSE), T2 FLAIR, conventional MRI followed by DWI, PWI, MRS and finally post-contrast T1 weighted images. Conventional MR images were obtained with T1 [spin echo (SE), TR 409/TE 8.4] and FSE T2 weighted (TR 400/TE 94) spin echo sequences. T2 FLAIR with TI of $900 \mathrm{~ms}$. DWI were acquired using single-shot echo-planar imaging (EPI) sequence at multiple levels. About 20 slices of $5 \mathrm{~mm}$ thickness were obtained (repetition time (TR) 3,000 ms echo time (TE) 89 $\mathrm{ms}, \mathrm{b}$ values of 0 and $1,000 \mathrm{~mm} 2 \mathrm{~s}-1$ )in three orthogonal directions. For susceptibility-based PWI, the transitory signal loss during the bolus passage was to be detected with a $\mathrm{T} 2 *$ weighted gradient EPI sequence (TR $1410 \mathrm{~ms}$, TE $30 \mathrm{~ms}$ ). About 50 dynamic scans with a time resolution of $1.0 \mathrm{~s}$ per image were performed after intravenous bolus injection of $10 \mathrm{ml} \mathrm{Gd}$ - DTPA at a flow rate of $5 \mathrm{ml} \mathrm{s}-1$ and a $20 \mathrm{ml}$ saline flush.

\section{Morphologic and Enhancement Criteria}

On MRI, variables like signal intensity of the tumour on T1 Characteristics (isointense/hypointense/hyperintense)was compared to normal grey matter),T2 characteristics (isointense/hypointense/ hyperintense), tumour margin(well defined, lobulated, mushrooming, ill defined), peritumoural band (absent/partial/complete) and extent of surrounding oedema. Perilesional oedema was graded as absent (0), less than the size of tumour(+) and more than the size of tumour(++),pattern of contrast enhancement (none/homogeneous/heterogeneous/ intense vessel like) the dural tail sign (absent/present) were analysed. Presence of calcifications were assessed by blooming in Susceptibility weighted imaging The report was written according to the set pattern and was entered in the proforma for analysis. The data were statistically analyzed. The final pathological diagnosis in all patients was confirmed by biopsy.

\section{Results}

6 tumours were located in the frontal convexity, 2 parasagittal, 2 falx, 2 parietal, and one each in the torcula, temporal and sphenoid wing. Tumour size ranged from 3 to $8 \mathrm{~cm}$ in maximum diameter.

On T1W MRI, 9 cases displayed heterogeneous signals with hypointensity, isointensity and hyperintensity, One lesion showed isointense signal, 5 were nonhomogeneously hypointense. On T2W MRI, 8 tumours were nonhomogeneouslyhyper intense, and two lesions showed homogeneously hyperintense signals. Isointensity was seen in one lesion. One of the lesions appeared non homogeneously hypointense. Heterogeneous intensity was seen in three cases. Peritumoural band showed a hypointense rim on T1W and a hyperintense rim on T2W. Presence of the peritumoural band was evaluated. Peritumoural band was completely present in one case, it was partially apparent in53\%tumours and absent in $40 \%$ of cases.

On post-contrast $\mathrm{T} 1 \mathrm{~W}$, all the tumours showed heterogeneous enhancement.50\% of the cases 


\section{JMSCR Vol||05||Issue||01||Page 15163-15168||January}

displayed oedema larger than the tumour. No Perifocal oedema was seen in one case. Seven cases showed oedema less than the size of the tumour.

Presence of the dural tail sign with thickened enhancing dura extending from the tumour on post-contrast T1W was also assessed. Dural tail sign was seen in two of the 15 tumours. Dural tail was absent in $86 \%$ of cases. Calcifications were noted in susceptibility weighted images in two cases. Calcifications were absent in $86 \%$ of the lesions. $60 \%$ of the tumours showed restriction in Diffusion weighted imaging. All the tumours showed increased perfusion.

Table 1. Table depicting patient characteristics

\begin{tabular}{|c|c|c|c|c|c|c|c|c|c|c|c|c|}
\hline No. & age & sex & location & $\mathrm{T} 1$ & $\mathrm{~T} 2$ & oedema & $\mathrm{CE}$ & $\begin{array}{c}\text { Dural } \\
\text { tail }\end{array}$ & $\begin{array}{l}\text { Peritumoural } \\
\text { band }\end{array}$ & Calcifn & $\begin{array}{c}\text { MRI } \\
\text { diagnosis }\end{array}$ & HPR \\
\hline 1 & 70 & $\mathrm{~F}$ & torcular & $\begin{array}{c}\text { Inhomo } \\
\text { hypo }\end{array}$ & hetero & + & hetero & - & partial & - & atypical & atypical \\
\hline 2 & 26 & $\mathrm{M}$ & $\begin{array}{l}\text { Sphenoid } \\
\text { wing }\end{array}$ & $\begin{array}{l}\text { Homo } \\
\text { iso }\end{array}$ & $\begin{array}{l}\text { Homo } \\
\text { iso }\end{array}$ & + & homo & - & complete & + & aypical & atypical \\
\hline 3 & 34 & M & falx & $\begin{array}{c}\text { Inhomo } \\
\text { hypo }\end{array}$ & hetero & ++ & hetero & - & absent & - & atypical & atypical \\
\hline 4 & 62 & $\mathrm{~F}$ & frontal & hetero & $\begin{array}{c}\text { Inhomo } \\
\text { hyper }\end{array}$ & + & hetero & + & partial & - & atypical & atypical \\
\hline 5 & 45 & $\mathrm{M}$ & frontal & $\begin{array}{c}\text { Inhomo } \\
\text { hypo }\end{array}$ & hypo & 0 & hetero & - & partial & - & typical & atypical \\
\hline 6 & 65 & $\mathrm{M}$ & frontal & hetero & $\begin{array}{c}\text { Inhomo } \\
\text { hyper }\end{array}$ & + & hetero & - & absent & - & atypical & atypical \\
\hline 7 & 68 & $\mathrm{~F}$ & frontal & hetero & $\begin{array}{c}\text { Inhomo } \\
\text { hyper }\end{array}$ & ++ & hetero & + & partial & - & atypical & atypical \\
\hline 8 & 46 & $\mathrm{~F}$ & frontal & $\begin{array}{c}\text { Inhomo } \\
\text { hypo }\end{array}$ & $\begin{array}{l}\text { Homo } \\
\text { hyper }\end{array}$ & ++ & hetero & - & partial & - & atypical & atypical \\
\hline 9 & 44 & $\mathrm{M}$ & parasagittal & $\begin{array}{c}\text { Inhomo } \\
\text { hypo }\end{array}$ & $\begin{array}{l}\text { Homo } \\
\text { hyper }\end{array}$ & + & hetero & - & partial & + & typical & atypical \\
\hline 10 & 55 & $\mathrm{M}$ & parasagittal & hetero & $\begin{array}{c}\text { Inhomo } \\
\text { hyper }\end{array}$ & ++ & hetero & - & absent & - & atypical & atypical \\
\hline 11 & 31 & $\mathrm{M}$ & parietal & hetero & $\begin{array}{c}\text { Inhomo } \\
\text { hyper }\end{array}$ & ++ & hetero & - & absent & - & atypical & atypical \\
\hline 12 & 76 & $\mathrm{~F}$ & falx & hetero & $\begin{array}{c}\text { Inhomo } \\
\text { hyper }\end{array}$ & + & hetero & - & absent & - & atypical & atypical \\
\hline 13 & 26 & $\mathrm{M}$ & parietal & hetero & $\begin{array}{l}\text { Inhom } \\
\text { hyper }\end{array}$ & + & hetero & - & partial & - & atypical & atypical \\
\hline 14 & 66 & $\mathrm{~F}$ & frontal & hetero & hetero & ++ & hetero & - & partial & - & atypical & atypical \\
\hline 15 & 25 & $\mathrm{M}$ & temporal & hetero & $\begin{array}{c}\text { Inhomo } \\
\text { hyper }\end{array}$ & ++ & hetero & - & absent & - & atypical & atypical \\
\hline
\end{tabular}




\section{JMSCR Vol||05||Issue||01||Page 15163-15168||January}

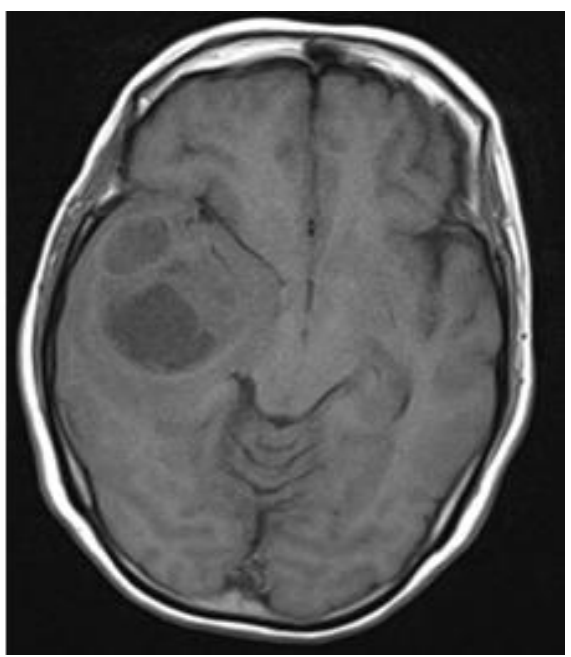

Fig.1. T1W MR image showing predominantly heterogeneous lesion with hypointense areas in the right temporal region

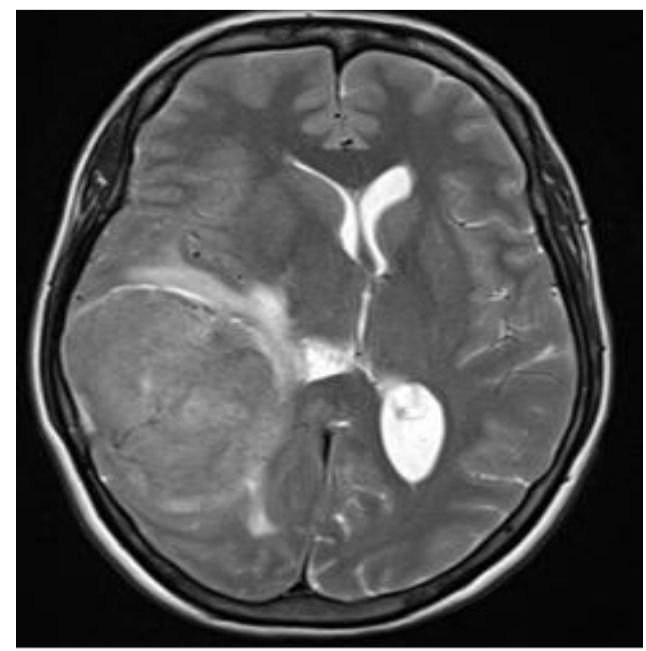

Fig.3. T2W image showing mildly hyperintense well circumscribed mass in the right temporoparietal region with perilesional edema.

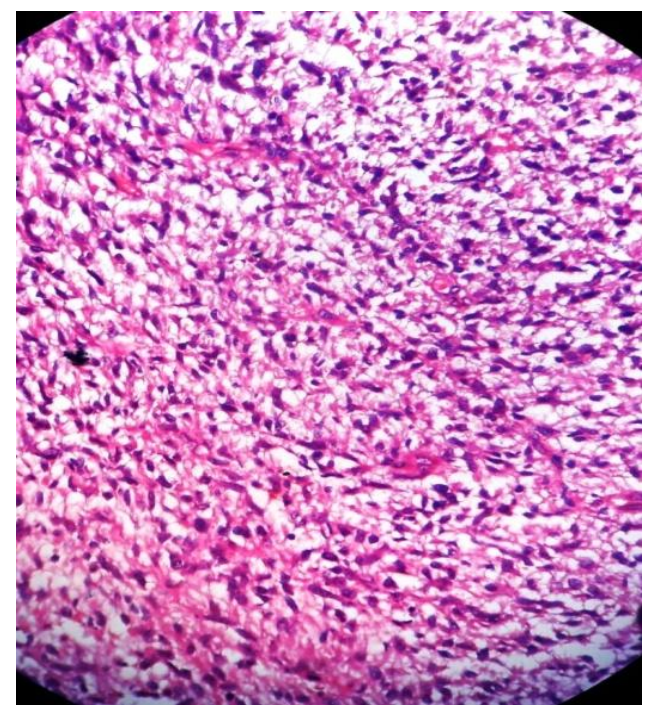

Fig. 5. A typical meningioma under low power microscope

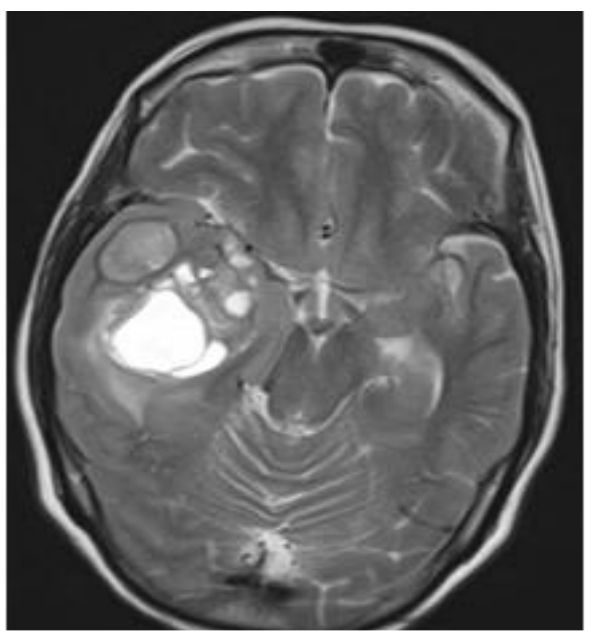

Fig.2. T2W image shows hyperintense lesion with cystic areas

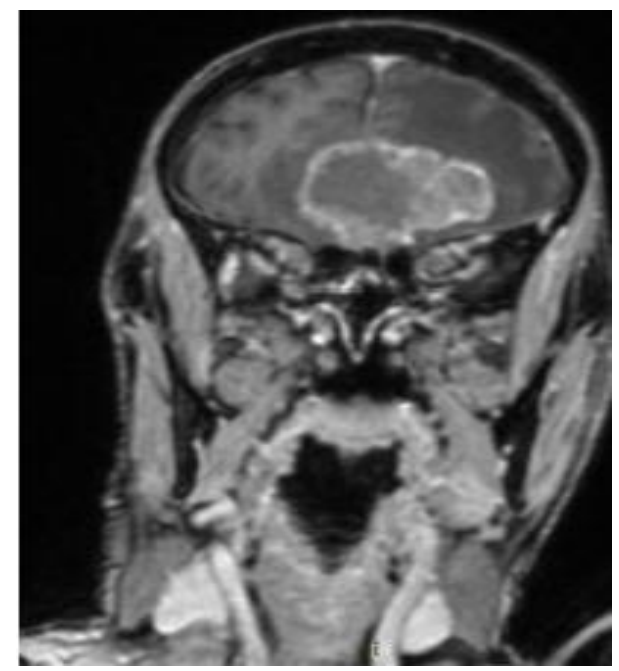

Fig. 4 Fat suppressed T1 post contrast image shows heterogenous enhancement.

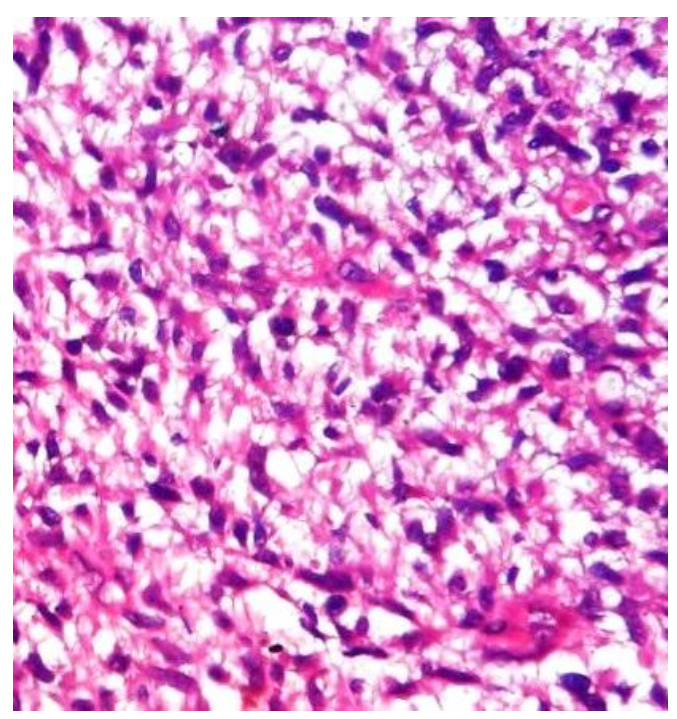

Fig. 6 .A typical meningioma under high power microscope 


\section{Discussion}

Even though majority of meningiomas can be diagnosed easily with MR imaging, the diagnosis can be challenging when meningiomas show atypical imaging findings. MRI plays important role in the diagnosis of meningioma. With conventional MR imaging the specificity of WHO Grade 1 meningiomas is almost $99 \%$. As the WHO grade 2 and 3 meningiomas show atypical features in conventional imaging the sensitivity is much lower compared to Grade 1 tumours.

On MRI, the tumour is isointense- or hypointense on non-contrast T1-W, and isointense- or hyperintense on T2-W. Homogeneous enhancement is observed after contrast administration in typical meningiomas. However $15 \%$ of all meningiomas can show cystic, necrotic, or fatty changes.

In the present study 3 of the cases showed heterogeneous appearance in $\mathrm{T} 1 \mathrm{~W}$ images. Cystic components, which can be partially necrotic, were seen in five of the cases. Fatty change was not seen. Only 3 of the cases displayed findings similar to benign meningiomas. All the cases demonstrated heterogeneous contrast enhancement.

Dural tail sign was seen in only two of these 15tumours. As summarized in the study of R.D Tien, P.J Yang, P.K Chuet $\mathrm{al}^{5}$ dural tail sign was a found in all typical meningiomas .Calcification was also found in two cases. A previous report considered the absence of calcification in malignant meningiomas. This may be associated with increased growth rate.

The T1 hypointense peri-tumoural band denotes the extra-axial nature of the tumour .A complete peri-tumoural band was seen in only one tumour. All the other tumours showed partial or complete disappearance of the peri-tumoural band.

In our study significant perilesional oedema was present surrounding most of the tumours. Perilesional oedema is not a feature of typical meningiomas. Perilesional oedema ${ }^{1}$ is seen with atypical and malignant varieties. However the amount of perilesional oedemadoes not correlate with the histological type.
$5-15 \%$ of meningiomas fall under WHO Grade II category. Histopathological criteria for diagnosis are either 4-19 mitotic figures/10 HPF orbrain invasion or three of the histologic features like increased cellularity, small cells with high N/C ratio, large and prominent nucleoli, pattern less or sheet like growth or loss of lobular architecture, or foci of spontaneous or geographic necrosis.

The accuracy of the study in detecting atypical lesions increases more when considering multiple parameters in conventional imaging When combined with advanced MRI techniques, including diffusion ${ }^{7}$ and perfusion weighted ${ }^{2}$ MRI and spectroscopy, the sensitivity and specificity positive predictive values reach almost upto $100 \%$ in differentiating benign and atypical and anaplastic meningiomas.

\section{Conclusion}

The present study attempted to predict the histologic nature of meningiomas to aid in surgical and treatment planning, because recurrence rate and prognosis in atypical meningiomas are different from those in benign meningiomas. If accurate grading of the tumours can be done prior to surgery, the surgeons can be more cautious in removing the tumours as completely as possible. Apart from the morphologic and enhancement characteristics MR imaging can identify the proximity to a vessel, dural and osseous involvement which are vital information while planning surgery.

\section{Acknowledgements}

Our sincere thanks to Dr Anil Peethambaran, Head of the Department of Neurosurgery, Govt. Medical College, Trivandrum and Dr, Krishna Balachandran, Head of the Department of Pathology, Govt. Medical College, Trivandrum.

\section{Conflict of Interest- none.}

\section{References}

1. Nakano T, Asanoa K, Miura H, Itoh S, Suzuki S (2002), Meningiomas with brain 
oedema: radiological characteristicson MRI and review of the literature. Clin Imaging 26:243-249

2. Cha S, Yang L, Johnson G, Lai A, Chen MH, Tihan T, Wendland M, Dillon WP (2006) Comparison of microvascular permeability measurements, K(trans), determined with conventional steady-state T1-weighted and firstpassT $2 *$-weighted MR imaging methods in gliomas and meningiomas. AJNR Am J Neuroradiol 27:409-417

3. J Jääskeläinen, M Haltia, A Servo. A typical and anaplastic meningiomas: radiology, surgery, radiotherapy, and outcome. SurgNeurol, 25 (1986), pp. 233-242

4. C.C Coke, B.W Corn, M Werner-Wasik, Y Xie, W.J Curran Jr.Atypical and malignant meningiomas: an outcome report of seventeen cases JNeurolOncol, 39 (1998), pp. $65-70$

5. R.D Tien, P.J Yang, P.K Chu'Dural tail sign' a specific MR sign of meningioma?.J Comput Assist Tomogr, 15 (1991), pp. 64-66

6. Y Tanaka, M Matsuo Role of MR imaging in the differentiation of benign and non benign intracranial meningiomas: the utility of contrast-enhanced T1-weighted images. Nippon ActaRadiol, 56 (1996), pp. $1-8$

7. C.G Filippi, M.A Edgar, A.M Ulug, J.C Prowda, L.A Heier, R.D Zimmerman. Appearance of meningiomas on diffusionweighted images: correlating diffusion constants with histopathologic findings. AJNR Am J Neuroradiol, 22 (2001), pp. $65-72$

8. Factors associated with recurrence of postoperative meningioma: a clinical study of 138 patients Alfotih Gobran, Fang Cheng Li, Xin Ke Xu, Shang Yi Zhang Romanian Neurosurgery (2013) XX 4: 379 $-387$. 